\title{
EVALUACIÓN DE LAS ACTITUDES DEL PROFESORADO RESPECTO A LOS TEMAS CTS: NUEVOS AVANCES METODOLÓGICOS
}

\author{
Manassero Mas, M. Antonia ${ }^{1}$; Vázquez Alonso, Ángel ${ }^{2}$ y Acevedo Díaz, José Antonio \\ ${ }^{1}$ Departamento de Psicología. Universidad de las Islas Baleares \\ ${ }^{2}$ Facultad de Ciencias de la Educación. Universidad de las Islas Baleares \\ ${ }^{3}$ Inspección de Educación. Consejería de Educación de la Junta de Andalucía. Delegación Provincial de Huelva \\ ma.manassero@uib.es \\ angel.vazquez@uib.es \\ ja_acevedo@vodafone.es
}

\begin{abstract}
Resumen. Este estudio presenta un análisis de las actitudes del profesorado de ciencias hacia los temas de ciencia, tecnología y sociedad (CTS) basados en la categorización del Cuestionario de Opiniones sobre CTS (COCTS) empleando nuevos procedimientos metodológicos. Éstos permiten aproximaciones más precisas al diagnóstico de las actitudes desde perspectivas cuantitativas y también cualitativas. Además el método es flexible, pues permite enfoques sobre objetos de actitud muy variados, como una cuestión singular, una dimensión CTS concreta que engloba varias cuestiones, diagnóstico de actitudes individuales y aplicaciones de estadística inferencial para contrastar hipótesis. La valoración global de los resultados confirma, con mayor profundidad y fiabilidad, la deficiente formación inicial en los temas CTS, a la vez que valida la nueva metodología y el progreso en la evaluación de estos temas. Finalmente, se aboga por una modificación de la formación del profesorado de ciencias, inicial y continua, que se ajuste más a las propuestas de innovación del movimiento CTS que inspiran las diversas reformas de la enseñanza de las ciencias en el mundo.

Palabras clave. Ciencia, tecnología, sociedad, pensamiento del profesorado, evaluación de actitudes, naturaleza de la ciencia.

Summary. Science teachers' attitudes and beliefs toward Science, Technology, Society (STS) topics are analysed applying new methodological procedures based on the categorization of the Questionnaire of Opinions on STS (QOSTS) statements. The new contributions and improvements allow more accurate and new quantitative and qualitative approaches. The method is flexible and allows the diagnosis of personal specific attitudes, assessing specific STS issues or dimensions (using a set of items), and also inferential statistical procedures to contrast hypothesis. The results confirm, with stronger depth and validity, teachers' faulty training in STS topics. On the other hand, results validate the new methodology and the progress in the evaluation of these topics. Finally, the results claim for a change of the initial and continuous science teacher training, to fulfil the innovative proposals of the STS movement, which inspire the different science educational reforms all over the world.
\end{abstract}

Keywords. Science, technology, society, teacher thinking, attitudinal assessment, nature of science.

\section{INTRODUCCIÓN}

Durante los últimos años, científicos, educadores y organizaciones internacionales de educación en ciencias convergen en el objetivo de que los estudiantes consigan desarrollar concepciones informadas y apropiadas sobre las relaciones mutuas entre la ciencia, la tecnología y la sociedad (CTS). Ahora bien, la investigación ha mostrado, y sigue mostrando, de forma consistente, que estudiantes y profesores no alcanzan una comprensión adecuada de las cuestiones que aborda la educación CTS en la enseñanza de las ciencias en nuestro contexto (Acevedo, 2001; Acevedo,
Vázquez, Acevedo y Manassero, 2002; Acevedo, Vázquez, Manassero y Acevedo, 2002a, 2002b; Manassero y Vázquez, 1998, 2001) y en otros países (Fleming, 1987, 1988; Ryan y Aikenhead, 1992), hecho que es, a la vez, un diagnóstico de la situación y un acicate para su mejora. Por ello, numerosos esfuerzos han sido y continúan siendo dirigidos para formar a los estudiantes y, especialmente, al profesorado de ciencias en estos temas (Acevedo, 1996; Akerson, Abd-El-Khalick y Lederman, 2000; Cho, 2002; Lederman, 1992; Shapiro, 1996; Solbes, Vilches y Gil, 2002). 
En el marco de la enseñanza de las ciencias, la evaluación de las creencias CTS de los estudiantes y el profesorado constituye un problema relevante de la investigación por la necesidad de conocer sus concepciones iniciales y los aprendizajes alcanzados; sin embargo, no es sencilla por dos razones principales. En primer lugar, por la naturaleza poliédrica y compleja de la empresa científico-tecnológica y sus relaciones con la sociedad y, en segundo lugar, por la propia dificultad inherente a la tarea, que debe afrontar diversos problemas metodológicos relativos a la validez y fiabilidad de los procedimientos e instrumentos de evaluación aplicados en la investigación. El carácter complejo y polémico de las creencias CTS surge en parte por la naturaleza provisional y la dinámica propia de estos temas interdisciplinares. Por ello, el acuerdo sobre las cuestiones CTS es limitado y está condicionado por factores culturales, coexistiendo discrepancias sobre los significados de ciertos temas CTS entre filósofos, historiadores, sociólogos y educadores en ciencias, que dificultan su transposición didáctica y su pertinencia para la enseñanza de la ciencia o la tecnología. A las dificultades metodológicas se dedicará un apartado específico más adelante.

\section{ACTITUDES, CREENCIAS Y OPINIONES}

En este estudio se usa el término actitud para referirse a las ideas sobre los temas CTS por considerarlo más válido teóricamente, y esta elección se justifica brevemente en este párrafo. A veces se tiene una visión muy reducida de la actitud, como una predisposición positiva o negativa limitada al aprendizaje (en tal caso sería más apropiado hablar de interés por aprender). Sin embargo, el concepto de actitud es más amplio, pues se refiere a disposiciones psicológicas personales que implican la valoración, positiva o negativa, de un objeto a través de respuestas explícitas o implícitas. Su uso para referirse a las ideas sobre los temas CTS pueda parecer extraño por su novedad en el área y, por ello, quizás requiere una justificación (Vázquez y Manassero, 1995). Habitualmente, la literatura especializada utiliza vocablos como actitudes, creencias, opiniones, concepciones, e incluso conocimientos, para referirse a ideas fuera del cuerpo estricto de conocimientos de la ciencia (p.e., las relaciones CTS), tal vez por transposición de la terminología desarrollada en la ingente investigación sobre concepciones alternativas. No obstante, puesto que se trata de constructos de naturaleza diferente, existen razones de peso para preferir el término actitud en vez de los anteriores.

Por un lado, a diferencia de los conocimientos científicos, los temas CTS están cargados de valores y son complejos, polifacéticos y cambiantes, por su propia naturaleza y por el estado actual de su investigación. En muchas cuestiones no existe suficiente consenso, y no se dispone de un cuerpo de conocimientos establecido universal que permita decidir unívocamente sobre la valoración de la respuesta a un tema concreto. En su lugar, hay acuerdos limitados en ciertos temas y conjeturas razonables sobre otros muchos, mientras también subsisten algunas áreas de clara controversia entre los especialistas. Por ello, cualquier idea sobre estas cuestiones no tiene un aval seguro, de modo que cada profesor decide su valoración sobre ellas, positiva o negativa, concepto más próximo a una actitud que a un conocimiento. Por otro lado, actualmente, el profesorado carece de una formación explícita sobre los temas CTS, pues hasta ahora no ha existido en España un plan específico y sistemático de formación del profesorado capaz de proporcionar un cuerpo de conocimientos CTS que sirva de referencia básica para ellos. Por tanto, también desde esta perspectiva parece excesivo considerar sus ideas como conocimientos o concepciones, sino que estarían más cerca de valoraciones u opciones personales.

Como se acaba de indicar, las cuestiones CTS están cargadas de valores, cuya gestión didáctica no requiere tanto de conocimientos específicos, sino más bien una posición de aceptar o rechazar, e incluso actuar, de acuerdo con la actitud que se acepta. El concepto de actitud que contiene a la vez elementos cognitivos, afectivos y de conducta se aproxima más que ningún otro a la naturaleza de los temas CTS porque engloba simultáneamente estas características. Los aspectos cognitivos (comprensión y conocimientos) son importantes, especialmente en los casos más polémicos, pero más esencial aún es la elección personal, la cual supone una valoración afectiva (mayor o menor grado de acuerdo o desacuerdo) sobre un tema CTS y que tiene una implicación global a través de los objetivos y finalidades educativos. Por ejemplo, de la ley de la gravedad cabe predicar su conocimiento, comprensión o aplicación y evaluar las concepciones alternativas o errores conceptuales respecto a las prescripciones del cuerpo de conocimientos científicos; sin embargo, respecto de la objetividad de la ciencia, la adhesión o el rechazo de la persona hacia ella resulta más relevante que su comprensión cognitiva, porque no existe un patrón universalmente aceptado.

En suma, el concepto de actitud, cuyo componente principal es la valoración afectiva del objeto más que su propio conocimiento, describe mejor el tipo de internalización de una persona ante las cuestiones CTS y la asimilación de ideas sin una formación reglada previa o en unas condiciones de gran complejidad y dinamismo del objeto, tal y como ocurre con los temas CTS. Por otra parte, la propuesta de utilizar la terminología de actitudes no sólo se sitúa en sintonía con la realizada por Shrigley y Koballa (1992) de mirar hacia los constructos ya elaborados y contrastados en otras áreas (las actitudes sería uno más), como respuesta a algunos problemas surgidos en la investigación en didáctica de las ciencias, en particular la psicología social, sino que supone un elemento de unificación conceptual en este tema.

\section{METODOLOGÍA E INSTRUMENTOS DE EVA- LUACIÓN CTS}

De acuerdo con lo apuntado más arriba, una de las cuestiones más importantes de la evaluación de actitudes CTS se refiere a las dificultades derivadas de los aspectos metodológicos implicados en la valoración de estos temas, que afectan sobre todo a la validez y fiabilidad de los instrumentos y los métodos aplicados y, en consecuencia, a la significación de los resultados obtenidos.

Durante los últimos cuarenta años se han desarrollado y usado numerosos cuestionarios de papel y lápiz para eva- 
luar las opiniones de los estudiantes (Manassero y Vázquez, 1998). Diversos estudios de revisión y crítica han puesto en evidencia numerosos fallos y defectos metodológicos encontrados en este tipo de investigaciones (Acevedo, Acevedo, Manassero y Vázquez, 2001; Gardner, 1996). Un resumen de las principales críticas podría ser el siguiente:

- La hipótesis de la percepción inmaculada, según la cual se supone que el investigador y la persona encuestada perciben y comprenden el texto de un cuestionario de la misma manera (Aikenhead y Ryan, 1992; Aikenhead, Ryan y Desautels, 1989; Lederman y O’Malley, 1990), de modo que el acuerdo o discrepancia con una frase siempre obedece a las mismas razones imaginadas por los diseñadores del instrumento.

- Los instrumentos reflejan en exceso los prejuicios de sus diseñadores, de manera que a través de las opciones ofrecidas a las personas se imponen, implícitamente, las propias creencias de los investigadores. Así, los resultados terminan atribuyendo a las personas investigadas unas opiniones que son más producto del instrumento aplicado que una representación fiel de las ideas de los propios encuestados.

- Los instrumentos normalizados limitan mucho la posibilidad de extraer conclusiones significativas y evaluar los cambios actitudinales, pues es difícil aclarar qué valor numérico de las puntuaciones corresponde a una «actitud adecuada o inadecuada», sobre todo por su escasa validez de contenido (correspondencia entre lo que se dice pretender medir y lo que realmente se mide), o por violar la unidimensionalidad de constructo, necesaria en cualquier instrumento para validar los resultados métricos y las interpretaciones consiguientes (Aikenhead, 1988; Shrigley y Koballa, 1992).

Puesto que la mayor parte de las investigaciones se había hecho con cuestionarios de papel y lápiz, estas críticas provocaron hace tiempo cierta prevención ante la investigación cuantitativa. Simultáneamente, esto coincidió con el auge de las metodologías cualitativas, más suaves y laxas (entrevistas, cuestionarios abiertos, estudios de caso, etc.), de manera que se produjo lentamente un cambio de paradigma metodológico. Aunque los métodos cualitativos se prestan a revelar mejor los procesos de pensamiento, también contribuyen a ocultar algunos sesgos y puntos críticos de la investigación; en particular, las interpretaciones del investigador respecto a los registros obtenidos (Lederman, 1992). Este dilema fue resuelto por Aikenhead (1988) comparando la validez de diversos instrumentos (escalas Likert, cuestionarios de elección múltiple, cuestionarios empíricamente desarrollados y entrevistas) y concluyendo que las entrevistas aportan mayor riqueza de datos pero consumen mucho tiempo, por lo que los cuestionarios empíricamente desarrollados a partir de preguntas abiertas y entrevistas previas constituyen una tercera vía muy valiosa, que combina las ventajas de los instrumentos cerrados con la riqueza de las entrevistas, ahorra mucho tiempo de aplicación y permite su aplicación a muestras grandes. El carácter empíricamente construido confiere una validez de contenido inherente a las cuestiones, que convierte al COCTS en un cuestionario atípico, donde añadir o eliminar frases (como si fuera un cuestionario de investigador) supone modificar su estructura y la naturaleza propia y, en consecuencia, la validez inherente al mismo (Aikenhead y Ryan, 1992; Ryan y Aikenhead, 1992). En particular, es interesante notar que el número de frases adecuadas, plausibles o ingenuas en cada cuestión depende de esta construcción empírica y no es un rasgo arbitrario controlado por el investigador, de modo que las potenciales objeciones como si fuera un cuestionario clásico, no tendrían razón de ser (Vázquez y Manassero, 1999).

En esta línea, se desarrollaron los instrumentos Views on Science, Technology and Society (VOSTS; Aikenhead y Ryan, 1989; Aikenhead, Ryan y Fleming, 1989) y Teacher's Belief about Science-Technology-Society (TBA-STS; Rubba y Harkness, 1993). Aunque el VOSTS se preparó originalmente para estudiantes de 16 y 17 años, también se ha usado con éxito con estudiantes universitarios y con profesores (Aikenhead y Ryan, 1992; Fleming, 1988; Zoller, Donn, Wild y Beckett, 1991a, 1991b). El TBA-STS fue elaborado para utilizarlo con el profesorado en formación inicial y en ejercicio. Partiendo de la taxonomía de actitudes relacionadas con la ciencia y la tecnología propuesta por Vázquez y Manassero (1995) y siguiendo pautas similares, las cuestiones de los dos instrumentos anteriores se han adaptado a nuestro contexto cultural, construyéndose así el Cuestionario de Opiniones sobre Ciencia, Tecnología y Sociedad (COCTS; Manassero y Vázquez, 1998; Manassero, Vázquez y Acevedo, 2001). La experiencia de aplicación del COCTS ha sugerido algunas nuevas líneas metodológicas básicas para mejorar aún más su validez y fiabilidad. Éstas son:

- Cambiar el modelo de respuesta única (porque ofrece una información mínima sobre el pensamiento de la persona encuestada) por otro de respuesta múltiple (que permite maximizar la información disponible en cada cuestión).

- Generar una nueva métrica que permita extraer de las respuestas múltiples toda la información que contienen.

- Definir unos índices actitudinales normalizados con un significado métrico invariante para todo el COCTS, que sintetice válidamente todo el conjunto de respuestas emitidas.

- Categorizar todas las frases que integran el COCTS mediante la valoración por un panel de jueces especialistas, que es la base de la aplicación de una métrica para calcular los índices actitudinales.

Una vez realizado este programa de mejoras metodológicas, que permiten tanto el análisis cualitativo como la aplicación cuantitativa de la estadística inferencial y la comparación de grupos en contextos de investigación (Manassero y Vázquez, 1999; Manassero, Vázquez y Acevedo, 2001), éstas deben aplicarse empíricamente para mostrar sus posibilidades y consolidar una línea de investigación fructífera y sistemática. El propósito de este estudio es presentar este ejemplo empírico de evaluación de las actitudes y creencias CTS de un grupo de profesores de ciencias en ejercicio empleando estos nuevos procedimientos metodológicos con el instrumento COCTS para mostrar sus ventajas y posibilidades. 


\section{ASPECTOS METODOLÓGICOS DE LA INVES- TIGACIÓN}

\section{Muestra}

Los participantes en esta investigación son profesores de secundaria en ejercicio, inscritos en un curso de formación en ejercicio sobre temas CTS en la educación científica, cuyas principales características figuran resumidas en la tabla I.

\section{Instrumento}

Las cuestiones aplicadas en este estudio se han extraído del mencionado COCTS, un banco de 100 ítems de opción múltiple (disponible en línea $<w w w . e t s . o r g>$ ) que permite a las personas encuestadas expresar sus propios puntos de vista en una amplia gama de temas CTS. Todas tienen el mismo formato de elección múltiple; se inicia con una cabecera de pocas líneas donde se plantea un problema respecto al cual se desea conocer la actitud de la persona encuestada, seguido de una lista de frases que ofrece un abanico de diferentes justificaciones sobre el tema planteado y, por último, tres opciones fijas que recogen diversas razones para no contestar, como «No entiendo la cuestión», «No sé lo suficiente sobre el tema para seleccionar una opción»y «Ninguna de las opciones satisface básicamente mi opinión». (Véase el texto de una cuestión en la tabla II.) Cada una de las frases alternativas fue clasificada por un panel de jueces como adecuada, plausible o ingenua según la cual se valoran las respuestas dadas por los encuestados, de acuerdo con el método descrito

Tabla I

Características de la muestra de profesorado participante en el estudio.

\begin{tabular}{|lccc|}
\hline Modalidad & & Frecuencia & \% Válido \\
\hline Biología & & 2 & 8,3 \\
Ingeniería & & 3 & 12,5 \\
Filosofía & & 3 & 12,5 \\
Física & & 2 & 8,3 \\
Matemáticas & & 1 & 4,2 \\
Química & & 13 & 54,2 \\
& Total & 24 & 100,0 \\
\hline Género & & & \\
Hombres & & 14 & 58,3 \\
Mujeres & & 10 & 41,7 \\
& Total & 24 & 100,0 \\
\hline Antigüedad & & & \\
$<5$ años & & 15 & 62,5 \\
5 a 23 años & & 9 & 37,5 \\
& Total & 24 & 100,0 \\
& Perdidos & 1 & \\
& & & \\
& Total & 25 & 100,0 \\
\hline
\end{tabular}

en el apartado «procedimiento». Un aspecto importante a de entrevistas, cuestionarios y respuestas abiertas de los estudiantes interpretados cualitativamente, de modo que las frases incluidas en sus ítems sintetizan las ideas de los estudiantes sobre cada tema (Aikenhead y Ryan, 1992).

Cada frase se ha codificado mediante un número (central) y dos letras (una inicial y otra final). El número central de cinco cifras se corresponde con el original del VOSTS y representa las distintas dimensiones, temas y subtemas de referencia. La letra anterior al número (A, P o I), representa la categoría (adecuada, plausible o ingenua) de la frase, según la clasificación realizada por los jueces. La letra final representa el lugar relativo que ocupa la frase dentro de cada cuestión, ordenándose alfabéticamente (A, B, C...). Los temas y subtemas CTS de las 18 cuestiones elegidas para este estudio constituyen una muestra que representa significativamente las dimensiones abordadas por el COCTS (Tabla III).

\section{Procedimiento}

Los datos de esta investigación se obtuvieron mediante la administración de las cuestiones seleccionadas al profesorado participante, que respondió libremente como una actividad de autoevaluación dirigida a explorar sus actitudes y creencias previas sobre los temas CTS.

Se ha utilizado un modelo de respuesta múltiple (MRM), donde quien responde al problema que se plantea valora su grado de acuerdo/desacuerdo con cada una de las frases alternativas existentes en cada cuestión sobre una escala de nueve puntos, para matizar mejor su valoración y facilitar la proporcionalidad métrica con las tres categorías de la categorización (Manassero y Vázquez, 1998; Vázquez y Manassero, 1999). Cada una de estas valoraciones directas otorgadas se transforman en un índice actitudinal (Tabla IV) según la clasificación en tres categorías (adecuada, plausible e ingenua) asignada previamente a cada frase por un panel de jueces expertos (Acevedo, Acevedo, Vázquez y Manassero, 2001; Manassero, Vázquez y Acevedo, 2001; Vázquez, Acevedo y Manassero, 2000). Esta clasificación en tres categorías fue sugerida por Aikenhead para operacionalizar los acuerdos de los especialistas -frases adecuadas e ingenuas- y los temas aún controvertidos -frases plausibles-(Rubba y Harkness, 1993).

La idea esencial del MRM es obtener índices actitudinales normalizados $(-1,+1)$ para todas las frases (dividiendo la valoración resultante de la escala por el rango), en función de la categoría asignada a cada una de ellas $(a / 4$ $p / 4 n / 4$ respectivamente). En esta escala de valoración, las frases adecuadas se valoran tanto más alto cuanto la puntuación dada por una persona se aproxime más al 9, las ingenuas cuanto más cercana esté al 1 y las plausibles (que incluyen aspectos parcialmente adecuados) cuanto más cercana esté al 5 (valor central de la escala).

Con el fin de mostrar el potencial del método para afrontar distintas evaluaciones actitudinales en investigación o en educación, las respuestas múltiples de la muestra de profesorado se analizarán siguiendo las distintas posibilidades señaladas. resaltar del COCTS es su construcción empírica, a partir 
Tabla II

Ejemplo de cuestión del COCTS. En la columna de la derecha se indica la categoría (adecuada [A], plausible [P] o ingenua [I]) correspondiente a cada opción.

\begin{tabular}{|c|c|c|c|c|c|c|c|c|c|c|c|}
\hline \multicolumn{11}{|c|}{$\begin{array}{l}10211 \text { Definir qué es la tecnología puede resultar difícil porque ésta sirve para muchas cosas, pero la tecnología } \\
\text { PRINCIPALMENTE es: }\end{array}$} & \multirow{3}{*}{ CAT } \\
\hline \multirow{2}{*}{\multicolumn{2}{|c|}{$\begin{array}{l}\text { Para cada una de las frases siguientes, marca el número de la escala que represente mejor } \\
\text { el grado de acuerdo entre tu propia opinión y la posición expuesta en la frase. }\end{array}$}} & \multicolumn{9}{|c|}{ Grado de acuerdo } & \\
\hline & & \multicolumn{3}{|c|}{ Bajo } & \multicolumn{3}{|c|}{ Medio } & \multicolumn{3}{|c|}{ Alto } & \\
\hline A. & Muy parecida a la ciencia. & 1 & 2 & 3 & 4 & 5 & 6 & 7 & 8 & 9 & $\mathrm{P}$ \\
\hline B. & La aplicación de la ciencia. & 1 & 2 & 3 & 4 & 5 & 6 & 7 & 8 & 9 & I \\
\hline C. & $\begin{array}{l}\text { Nuevos procesos, instrumentos, maquinaria, herramientas, aplicaciones, artilugios, } \\
\text { ordenadores o aparatos prácticos para el uso de cada día. }\end{array}$ & 1 & 2 & 3 & 4 & 5 & 6 & 7 & 8 & 9 & $\mathrm{P}$ \\
\hline D. & Robots, electrónica, ordenadores, sistemas de comunicación, automatismos, máquinas. & 1 & 2 & 3 & 4 & 5 & 6 & 7 & 8 & 9 & $\mathrm{P}$ \\
\hline E. & Una técnica para construir cosas o una forma de resolver problemas prácticos. & 1 & 2 & 3 & 4 & 5 & 6 & 7 & 8 & 9 & $\mathrm{P}$ \\
\hline F. & $\begin{array}{l}\text { Inventar, diseñar y probar cosas (p. e., corazones artificiales, ordenadores y vehículos } \\
\text { espaciales). }\end{array}$ & 1 & 2 & 3 & 4 & 5 & 6 & 7 & 8 & 9 & $P$ \\
\hline G. & $\begin{array}{l}\text { Ideas y técnicas para diseñar y hacer cosas; para organizar a los trabajadores, la gente de } \\
\text { negocios y los consumidores; y para el progreso de la sociedad. }\end{array}$ & 1 & 2 & 3 & 4 & 5 & 6 & 7 & 8 & 9 & A \\
\hline H. & Saber cómo hacer cosas (p. e., instrumentos, maquinaria, aparatos). & 1 & 2 & 3 & 4 & 5 & 6 & 7 & 8 & 9 & $P$ \\
\hline \multicolumn{11}{|c|}{ Si alguna de las frases siguientes es aplicable a las opciones anteriores, escribe la letra de la opción a su lado. } & \\
\hline 1. & No lo entiendo. & & & & & & & & & & \\
\hline 2. & No sé lo suficiente sobre este tema para elegir una opción. & & & & & & & & & & \\
\hline 3. & Ninguna de estas opciones satisface básicamente mi opinión. & & & & & & & & & & \\
\hline
\end{tabular}

Tabla III

Tabla de especificaciones de los temas abordados en las cuestiones del COCTS aplicadas en este estudio con los números de referencia originales del COCTS completo (los que no aparecen corresponden a temas sin representante en esta muestra).

\begin{tabular}{|c|c|c|}
\hline Temas & Subtemas & Ítem \\
\hline \multicolumn{3}{|c|}{ Definiciones } \\
\hline \multirow{3}{*}{ 1. Ciencia y tecnología } & 01. Ciencia & 10111 \\
\hline & 02. Tecnología & 10211 \\
\hline & 04. Interdependencia & $10412^{*}, 10413^{*}$ \\
\hline \multicolumn{3}{|c|}{ Sociología externa de la ciencia } \\
\hline \multirow{4}{*}{ 2. Influencia de la sociedad sobre la ciencia/tecnología } & 04. Ética & 20411 \\
\hline & 05. Instituciones educativas & 20511 \\
\hline & 06. Grupos de interés especial & 20611 \\
\hline & 08. Influencia general & $20811^{*}, 20821^{*}$ \\
\hline \multirow{2}{*}{ 4. Influencia de la ciencia/tecnología sobre la sociedad } & 01. Responsabilidad social & 40111 \\
\hline & 02. Decisiones sociales & 40211 \\
\hline \multicolumn{3}{|c|}{ Sociología interna de la ciencia } \\
\hline \multirow{2}{*}{ 6. Características de los científicos } & 01. Motivaciones & 60111 \\
\hline & 05. Efectos de género & 60511 \\
\hline 7. Construcción social del conocimiento científico & 02. Decisiones científicas & 70211 \\
\hline \multicolumn{3}{|c|}{ Epistemología } \\
\hline \multirow{4}{*}{ 9. Naturaleza del conocimiento científico } & 02. Modelos científicos & 90211 \\
\hline & 05. Hipótesis, teorías y leyes & 90511 \\
\hline & 06. Aproximación a las investigaciones & 90611 \\
\hline & 10. Estatus epistemológico & 91011 \\
\hline
\end{tabular}


Tabla IV

Modelo de respuesta múltiple para una cuestión del COCTS. Significado de las puntuaciones directas de acuerdo/desacuerdo con cada frase alternativa, asignaciones de puntos en la escala de valoración y los procedimientos de cálculo de los índices actitudinales desde las puntuaciones directas.

\begin{tabular}{|c|c|c|c|c|c|c|c|c|c|c|c|c|c|c|c|c|}
\hline \multirow{2}{*}{$\begin{array}{r}\text { Categorías } \\
\text { Escala }\end{array}$} & \multirow{2}{*}{$\begin{array}{l}\begin{array}{c}\text { Número } \\
\text { de }\end{array} \\
\text { posiciones }\end{array}$} & \multicolumn{9}{|c|}{$\begin{array}{l}\text { Escala de valoración: } \\
\text { transformación de las puntuaciones directas }\end{array}$} & \multicolumn{3}{|c|}{$\begin{array}{c}\text { Puntuaciones } \\
\text { actitudinales directas }\end{array}$} & \multicolumn{3}{|c|}{$\begin{array}{l}\text { Índices de actitud } \\
\text { de categoría }\end{array}$} \\
\hline & & 9 & 8 & 7 & 6 & 5 & 4 & 3 & 2 & 1 & Máx. & Fórmula & Mín. & Máx. & Fórmula & Mín. \\
\hline \multicolumn{2}{|c|}{ Grado de acuerdo } & Total & $\begin{array}{l}\text { Casi } \\
\text { total }\end{array}$ & Alto & $\begin{array}{c}\text { Parcial } \\
\text { alto }\end{array}$ & Parcial & $\begin{array}{c}\text { Parcial } \\
\text { bajo }\end{array}$ & Bajo & $\begin{array}{l}\text { Casi } \\
\text { nulo }\end{array}$ & Nulo & & & & & & \\
\hline Adecuadas & $\mathrm{N}_{\mathrm{a}}$ & 4 & 3 & 2 & 1 & 0 & -1 & -2 & -3 & -4 & $+4 \mathrm{~N}_{\mathrm{a}}$ & $\Sigma a_{j}$ & $-4 \mathrm{~N}_{\mathrm{a}}$ & +1 & $\mathrm{I}_{\mathrm{a}}=\Sigma \mathrm{a}_{\mathrm{j}} / 4 \mathrm{~N}_{\mathrm{a}}$ & -1 \\
\hline Plausibles & $\mathrm{N}_{\mathrm{p}}$ & -2 & -1 & 0 & 1 & 2 & 1 & 0 & -1 & -2 & $+2 \mathrm{~N}_{\mathrm{p}}$ & $\Sigma p_{j}$ & $-2 N_{p}$ & +1 & $I_{p}=\Sigma p_{j} / 2 N_{p}$ & -1 \\
\hline Ingenuas & $\mathrm{N}_{\mathrm{n}}$ & -4 & -3 & -2 & -1 & 0 & 1 & 2 & 3 & 4 & $+4 \mathrm{~N}_{\mathrm{n}}$ & $\sum n_{j}$ & $-4 N_{n}$ & +1 & $I_{n}=\sum n_{j} / 4 N_{n}$ & -1 \\
\hline Total & $\mathrm{N}$ & & & & & & & & & & Índice & de actitud & global & 1 & $\mathrm{I}=\left(\mathrm{I}_{\mathrm{a}}+\mathrm{I}_{\mathrm{p}}+\mathrm{I}_{\mathrm{n}}\right) / 3$ & -1 \\
\hline
\end{tabular}

$a_{j}$ : puntuación de valoración directa para la frase «adecuada» $\mathrm{j}$ $p_{i}$ : puntuación de valoración directa para la frase «plausible» $\mathrm{j}$

$n_{j}$ : puntuación de valoración directa para la frase «ingenua» $\mathrm{j}$

$\Sigma$ : suma las puntuaciones directas desde $j=1$ a $j=N_{a}\left(j=N_{p}\right.$ o $\left.j=N_{n}\right)$ para el conjunto de las frases pertenecientes a cada una de las categorías «adecuadas», «plausibles» $\mathrm{o}$ «ingenuas»

\section{RESULTADOS}

Los parámetros utilizados para realizar una primera aproximación a la descripción estadística son el índice actitudinal mínimo y máximo, la media y la desviación típica de cada frase para la muestra. En conjunto, el análisis de las medias de cada una de las frases para toda la muestra (no mostrada aquí por su excesiva extensión) revela un sesgo de la distribución hacia valores actitudinales positivos, pues los límites inferiores de las cuestiones que no llegan al mínimo (-1) son mucho más frecuentes que los límites superiores que no llegan al máximo (+1). Además, las puntuaciones más altas de los índices medios alcanzan algunos valores superiores a $+0,80$, mientras que las más bajas no son inferiores a -0,50, confirmándose también así el sesgo positivo de las actitudes CTS del profesorado. Este sesgo es un resultado esperable, en principio, aunque queda pendiente valorar si la magnitud del sesgo positivo es suficiente para valorar globalmente las actitudes de los estudiantes (Tabla V).

\section{PARÁMETROS GLOBALES DEL GRUPO}

Los parámetros descriptivos de los índices medios de cada uno de los grupos de frases adecuadas, plausibles e ingenuas muestran notables diferencias entre sí. Los índices de actitud son más altos y positivos en las frases adecuadas e ingenuas y ligeramente negativos en las frases plausibles. Esto indica que la contribución de las tres categorías al índice actitudinal global es diferente. Mientras que las frases adecuadas e ingenuas contribuyen de forma moderadamente positiva, las frases plausibles contribuyen muy poco, aunque con un tinte ligeramente negativo; con otras palabras, los profesores encuestados tienen más facilidad para identificar frases adecuadas, un poco menos para las ingenuas y todavía menos para las plausibles. Este patrón sugiere que la adhesión a las posiciones más definidas (adecuadas e ingenuas) de las cuestiones CTS planteadas parece relativamente más sencilla, pero es más difícil respecto a las frases más ambiguas (plausibles o parcialmente adecuadas). Este resultado tiene importantes consecuencias para la validez de los instrumentos de evaluación de actitudes usuales, constituidos por una serie de frases descontextualizadas (tipo Likert, p. e.), porque demuestra que la definición de las actitudes de las personas encuestadas pueden verse menoscabadas en función del tipo de frase elegida para representar un determinado tema, especialmente si no se corresponden con una posición totalmente aceptada, como es el caso de las frases plausibles. Este resultado también explica el enorme riesgo de falta de validez asociada inherentemente a las medidas que evalúan actitudes o creencias con sólo uno o dos ítems, donde la contribución de éstos es aún más crítica (Tabla VI). 
Tabla V

Ejemplificación de la estadística descriptiva en toda la muestra de los índices de cada frase alternativa para una de las cuestiones aplicadas (10111) y para algunas frases alternativas cuyo índice medio resulta extremo, muy alto o muy bajo (en negrita).

\begin{tabular}{|c|c|c|c|c|c|}
\hline Frases & $\mathbf{N}$ & Mínimo & Máximo & Media & Desviación est. \\
\hline P10211A & 24 & $-1,00$ & 1,00 &, 0625 & ,68067 \\
\hline I10211B & 25 & $-1,00$ &, 50 &,- 4900 & ,45917 \\
\hline P10211C & 25 & $-1,00$ & 1,00 &, 0600 & 68191 \\
\hline P10211D & 24 & $-1,00$ & 1,00 &, 1250 & ,64690 \\
\hline P10211E & 25 & $-1,00$ & 1,00 &,- 0600 & 65064 \\
\hline $\mathrm{P} 10211 \mathrm{~F}$ & 25 & $-1,00$ & 1,00 &,- 1000 & ,67700 \\
\hline A10211G & 25 & $-1,00$ & 1,00 & ,3000 & ,54962 \\
\hline $\mathrm{P} 10211 \mathrm{H}$ & 24 & $-1,00$ & 1,00 & ,2917 &, 55003 \\
\hline I10211B & 25 & $-1,00$ &, 50 &,- 4900 & 45917 \\
\hline I10412A & 24 &,- 50 & 1,00 &, 7813 & ,37090 \\
\hline I10412B & 25 & $-1,00$ &, 75 &,- 4700 & ,47500 \\
\hline P10412C & 25 & $-1,00$ & 1,00 &,- 4400 &, 56495 \\
\hline P20811B & 25 & $-1,00$ &, 50 &,- 4400 & ,58310 \\
\hline P60511A & 25 & $-1,00$ & 1,00 &,- 4400 & 61779 \\
\hline I60511I & 25 &, 25 & 1,00 & ,8800 & ,22958 \\
\hline I60511J & 25 &, 00 & 1,00 & ,8200 & ,29333 \\
\hline I90211A & 24 &, 00 & 1,00 & ,7292 & ,31205 \\
\hline
\end{tabular}

Tabla VI

Parámetros actitudinales estadísticos de la distribución de la media de los índices actitudinales estandarizados de cada profesor (-1, +1), correspondientes a las respuestas al conjunto de cuestiones del COCTS.

\begin{tabular}{|lccccc|}
\hline & Media & Dv. est. & Rango & Máximo & Mínimo \\
\hline Media puntuaciones $^{\mathbf{0}}$ & 0,096 & 0,136 & 0,526 & 0,301 & $-0,225$ \\
Adecuadas $^{\mathbf{a}}$ & 0,244 & 0,141 & 0,570 & 0,500 & $-0,070$ \\
Plausibles $^{\mathbf{a}}$ & $-0,006$ & 0,235 & 0,898 & 0,398 & $-0,500$ \\
Ingenuas $^{\mathbf{a}}$ & 0,156 & 0,222 & 0,803 & 0,676 & $-0,128$ \\
Índice global ponderado* $^{\text {Ind }}$ & 0,131 & 0,100 & 0,411 & 0,292 & $-0,119$ \\
\hline
\end{tabular}

o: promedio de las puntuaciones medias individuales de cada frase

a: promedio de las medias de los índices actitudinales estandarizados de las frases adecuadas, plausibles e ingenuas, respectivamente, de cada profesor

*: promedio de las medias de los tres anteriores (adecuadas, plausibles, ingenuas) 


\section{DIAGNÓSTICO DE ACTITUDES HACIA UNA CUESTIÓN}

Partiendo de los índices de actitud normalizados obtenidos para cada frase, se puede calcular un índice global para cada cuestión, que representa la actitud hacia esa cuestión. Para hacer este cálculo se podrían usar dos vías. Una sería calcular la media aritmética de los índices del conjunto de las frases en cada cuestión (índice de actitud global medio). La otra vía, un poco más sofisticada, calcularía primero, por separado, el promedio correspondiente a las frases adecuadas, plausibles e ingenuas, con lo cual se obtendrían tres índices (uno por cada categoría) y después se calcula el promedio de éstos (índice de actitud global ponderado); éste es el procedimiento ilustrado en la parte derecha de la tabla IV.

Como ejemplo de una y otra vía, se resume en la tabla VII el cálculo de los índices correspondientes a tres cuestiones; una con un gran número de frases plausibles (10211), otra con un gran número de frases ingenuas (90611) y otra más equilibrada (91011). Es posible realizar estudios microcualitativos en cuestiones concretas, analizando el índice medio de cada alternativa, que proporciona información directa de la actitud global de la muestra (o de cada persona) respecto a la creencia expresada en la frase. Además, los índices de las tres categorías adecuadas, plausibles e ingenuas suponen una buena aproximación al pensamiento de la muestra (o de cada individuo) sobre los puntos fuertes y débiles relacionados con las ideas más ingenuas o más adecuadas de la cuestión o tema. Por ejemplo, los resultados muestran que un punto fuerte de la actitud del grupo de profesores es identificar positivamente algunas creencias ingenuas sobre el método científico, pero falla respecto a las ideas más adecuadas y plausibles sobre éste, lo que contribuye negativamente a la actitud en esa cuestión (90611). Por el contrario, la actitud hacia el carácter de descubrimiento o de invención de las teorías científicas (91011) tiene un perfil opuesto, siendo ahora el punto más fuerte la identificación positiva de las alternativas adecuadas y plausibles, mientras que el más débil es el relacionado con las ideas ingenuas.

El análisis minucioso de las frases individuales también puede mostrar otros detalles más precisos sobre las valoraciones; por ejemplo, en la categoría ingenua de la cuestión 91011, aunque el promedio es prácticamente cero, hay diferencias importantes entre dos de las tres frases ingenuas, una nítidamente positiva y otra claramente negativa, de modo que este análisis también permite precisar aún más las carencias y aciertos del grupo.

La línea de trabajo esbozada en los párrafos anteriores con base en los índices cuantitativos sería susceptible de complementarse mediante análisis cualitativos que interpreten las respuestas a la luz del análisis interpretativo basado en el texto de cada frase. Por otro lado, desde una perspectiva docente, el valor formativo de este tipo de evaluación es valioso. Con base en los resultados de las dos cuestiones anteriores, la planificación de la enseñanza requeriría actuaciones de signo distinto en uno y otro tema, pues, mientras que en el primer caso quizás debería centrarse en la construcción de una idea más adecuada del método científico (débil en los resultados) a partir de las creencias ingenuas (aspecto más fuerte en los resultados), en el segundo caso, como la actitud más adecuada es el punto más fuerte, aunque coexistiendo con una cierta aceptación de ideas ingenuas, tal vez se requeriría una metodología de conflicto entre ambos tipos de creencias. En todo caso, la evaluación realizada suministra datos que permiten una reflexión formativa para la práctica educativa.

\section{DIAGNÓSTICO DE ACTITUDES HACIA UN TEMA}

Además de microanálisis para cada cuestión como los que se acaban de reseñar, también es posible realizar un estudio de dimensiones específicas mediante el análisis de un conjunto de cuestiones referentes a un mismo tema. De esta manera, en la dimensión CTS elegida (p. e., la naturaleza de la ciencia), se puede evaluar la actitud global, diagnosticar los puntos fuertes y débiles, evaluar las mejoras obtenidas después de la realización de un curso de formación en CTS o hacer comparaciones entre grupos. Como se requiere el uso de un conjunto de cuestiones pertenecientes a la dimensión seleccionada, en este caso el tratamiento cuantitativo de los índices actitudinales permite globalizar los resultados, de acuerdo con lo expuesto en párrafos precedentes.

Los índices globales de la tabla VIII indican que las actitudes son diferentes según el aspecto concreto abordado en cada cuestión. Mientras que en las cuestiones 90211 (epistemología de los modelos científicos) y 91011 (estatus del conocimiento científico) las actitudes son algo positivas, la 90611 (naturaleza del método científico) refleja una actitud negativa y la 90511 (relaciones entre hipótesis, leyes y teorías) tiene una actitud indecisa, ni positiva ni negativa. Los índices por categorías también muestran los puntos fuertes y débiles en cada cuestión. Las cuestiones 90211 y 91011 tienen índices de las distintas categorías bastante equilibrados entre sí, sin grandes diferencias entre ellos. Sin embargo, la cuestión 90611 tiene índices negativos en las categorías de frases adecuadas y plausibles, mientras que es positivo para las ingenuas; este último diagnóstico sugiere capacidad del profesorado para identificar los aspectos más ingenuos de la cuestión, pero dificultades notables para identificar los aspectos más adecuados de la misma, por lo que la conclusión sería mejorar y actualizar la formación en el tema concreto.

\section{DIAGNÓSTICO DE ACTITUDES PERSONALES}

La cuantificación de las respuestas de cada persona mediante los distintos índices actitudinales facilita los diagnósticos personalizados. El diagnóstico individual puede hacerse desde dos niveles claramente definidos:

- Relativo, que es la actitud de un individuo en comparazación con la de los demás componentes del grupo. La referencia podría ser el promedio de todo el grupo (para mejorar la fiabilidad en muestras pequeñas, debería excluirse la persona que se compara). 
Tabla VII

Valores de los índices correspondientes a tres cuestiones, una con un gran número de frases plausibles (10211), otra con un gran número de frases ingenuas (90611) y otra más equilibrada (91011), calculados por las dos vías, como media aritmética de las puntuaciones de todas las frases de cada cuestión o índice de actitud global promedio y como media de los índices de actitud de las tres categorías o índice de actitud global ponderado.

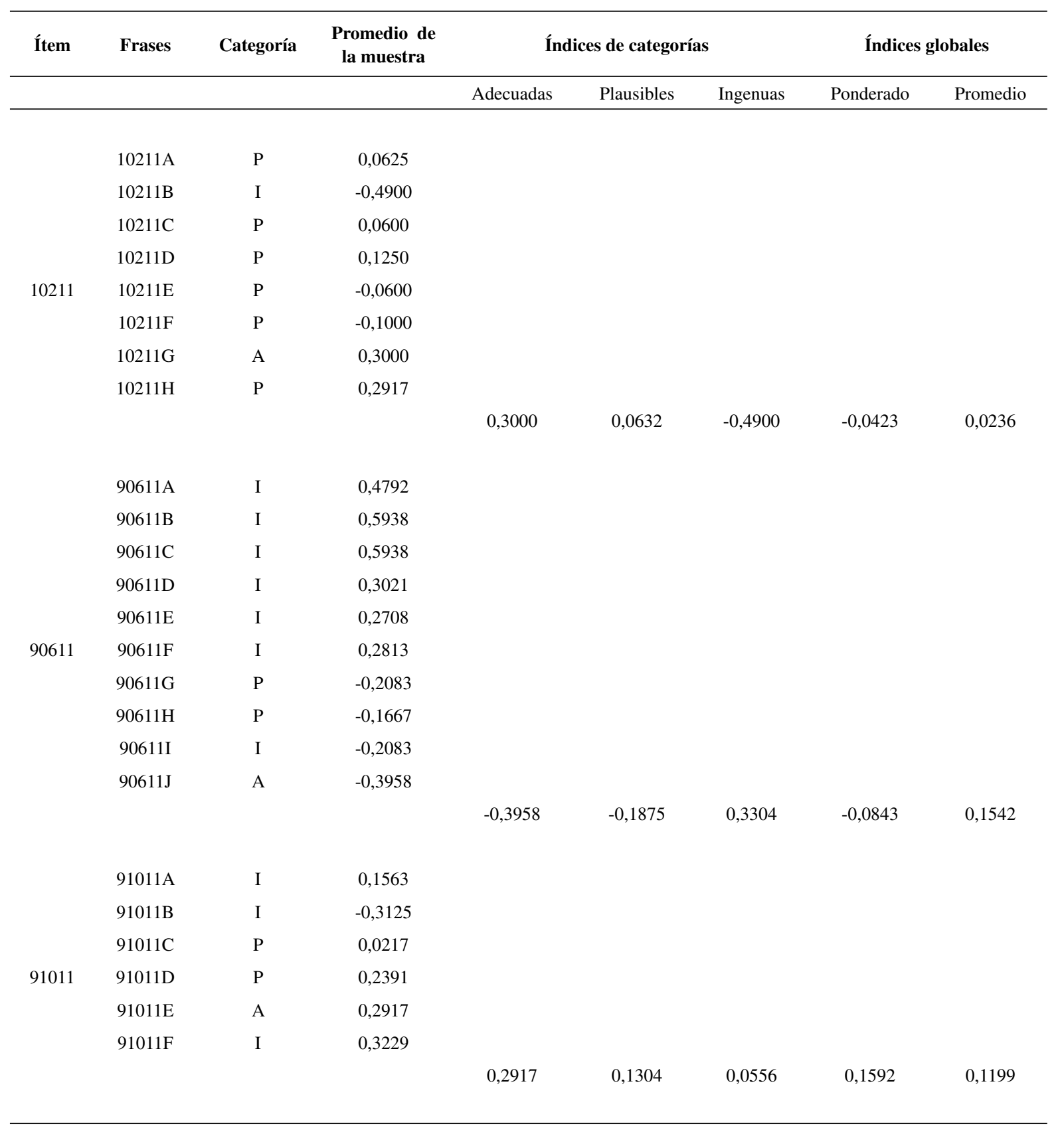


Tabla VIII

Índices actitudinales para las cuestiones representativas del tema «Naturaleza de la ciencia».

\begin{tabular}{|clcccc|}
\hline Ítem & \multicolumn{1}{c}{ Tema } & Adecuadas & Plausibles & Ingenuas & Índice global ponderado \\
\hline $\mathbf{9 0 2 1 1}$ & Modelos científicos & 0,2604 & 0,1610 & 0,2397 & 0,2204 \\
$\mathbf{9 0 5 1 1}$ & Hipótesis, teorías, leyes & $-0,0217$ & & 0,0230 & 0,0006 \\
$\mathbf{9 0 6 1 1}$ & Método científico & $-0,3958$ & $-0,1875$ & 0,3304 & $-0,0843$ \\
$\mathbf{9 1 0 1 1}$ & Descubrir o inventar & 0,2917 & 0,1304 & 0,0556 & 0,1592 \\
\hline
\end{tabular}

- Absoluto, que es la actitud del individuo respecto al perfil determinado por la categoría asignada a las frases por los jueces. La referencia sería esta categoría, representada por el perfil actitudinal óptimo +1 .

En conjunto, lo primero que salta a la vista es la diversidad de las actitudes individuales (Fig. 1). Los índices de los profesores cubren casi todo el espacio topológico de puntuaciones posibles en todas las frases alternativas. En este caso, la pluralidad de respuestas y actitudes puede ser un indicador de confusión y formación defectuosa del profesorado en el tema.

El diagnóstico individual facilita la planificación de las actuaciones didácticas de formación, orientadas a la mejora o al cambio de actitudes en cuestiones CTS. La complejidad de los procesos de cambio didáctico y del cambio de actitudes es de todos conocida y no es el objetivo de este artículo, pero desde la perspectiva que formación, desarrollo y cambio no son cuestiones independientes sino, tal vez, aspectos distintos de la misma realidad (Mellado, 2003). Las actitudes CTS del grupo relativamente reducido de profesores encuestados sugieren que dentro del grupo coexisten personas que defienden todas las posiciones actitudinales, de modo que una eficaz acción formativa, en este caso, debería ser muy personalizada; esto es, adaptada a las necesidades específicas de cada persona, basada en sus puntos fuertes y dirigida a mejorar los más débiles.

La precisión del diagnóstico individual combinada con la pluralidad de actitudes obtenidas - unidas al hecho de que la investigación que uno de los factores más poderosos en el cambio de actitudes muestra es la influencia del grupo de iguales- sugiere la posibilidad de organizar grupos de trabajo entre iguales como instrumento de cambio y mejora. La evaluación individual permite identificar las ideas más fuertes y más débiles, y también las personas que tienen las actitudes más adecuadas en cada frase, cuestión o dimensión; estas personas, que tienen a la vez actitudes adecuadas y la condición de iguales a quienes aprenden, podrían actuar como catalizadores del aprendizaje de los demás, en aquellos aspectos que son sus puntos fuertes, en un contexto de discusión o análisis en grupo, a modo de comunidades de aprendizaje, como un proceso interno de crecimiento personal y desarrollo profesional. Como complemento, se podrían utilizar también lecturas apropiadas sobre epistemología e historia y sociología de la ciencia, como base del trabajo de discusión o como información para tomar conciencia de las inconsistencias entre las ideas adecuadas que se sostienen y otras menos apropiadas, de modo que se pueda contribuir al cambio actitudinal desde la reflexión.

Diagnóstico individual: método científico

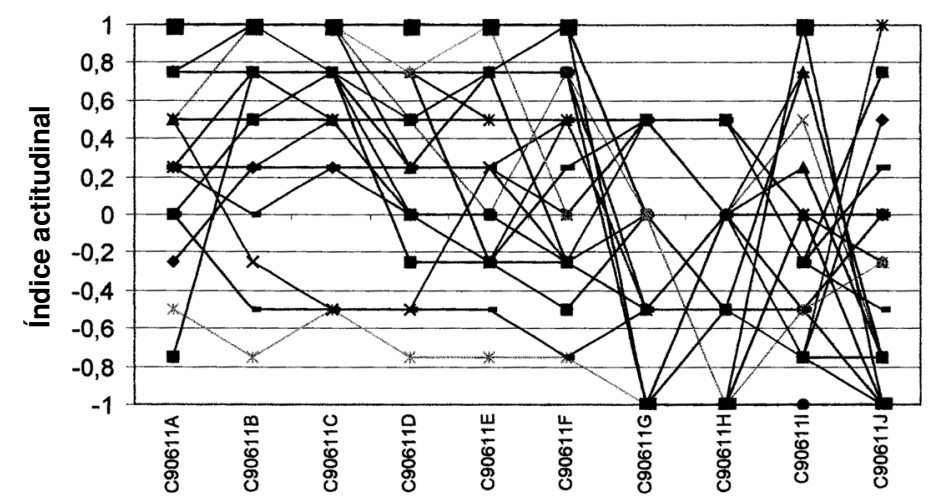


Figura 2

Representación gráfica conjunta de tres perfiles actitudinales individuales (el de mejor índice global, el peor y la media del grupo) sobre las diez alternativas (A, B, C... J) de la cuestión 90611.

\section{Diagnóstico individual: método científico}

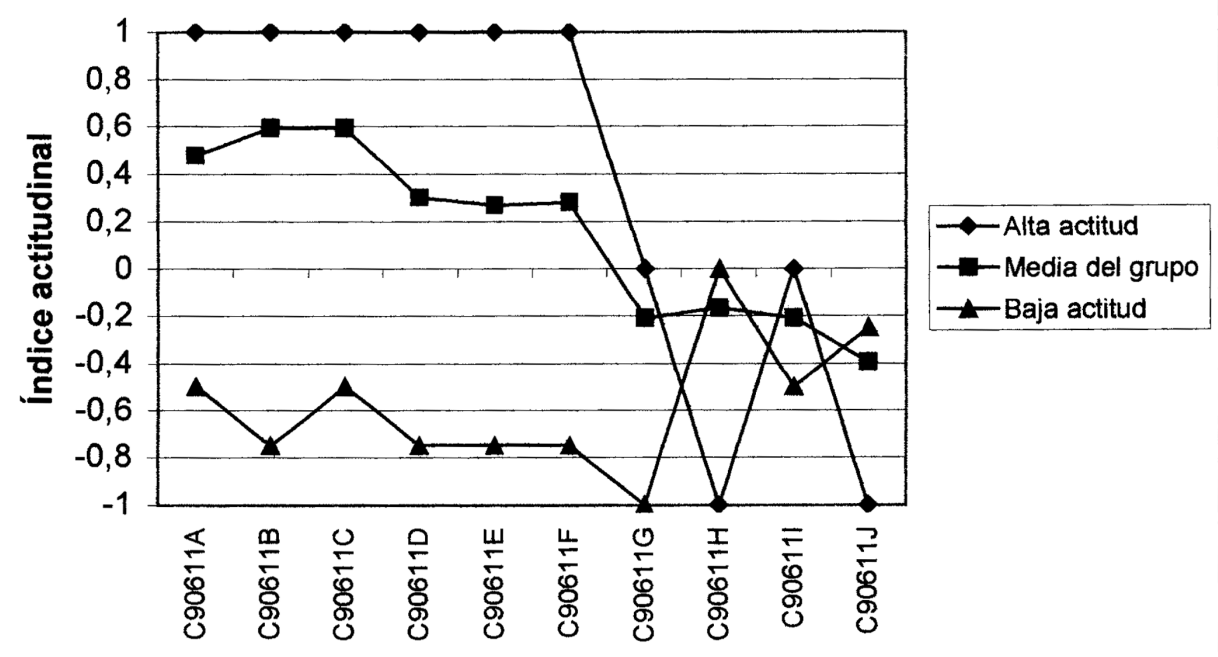

La figura 2 muestra los perfiles individuales de la persona con el índice de actitud más alto en esta cuestión (90611), con el índice más bajo y el promedio de todo el grupo. En este ejemplo, la persona con mejor actitud es también una referencia importante en las seis primeras alternativas de la cuestión, donde obtiene la máxima puntuación y, por tanto, podría ser utilizado como catalizador del grupo ante sus compañeros, para intentar promover el cambio actitudinal ante quienes necesitan mejorar su perfil; por el contrario, en otras alternativas quizás debería ser ayudada por las demás.

El diagnóstico personal cuantitativo ofrece indicadores que permiten llevar a cabo análisis cualitativos de interpretación y conexión entre las diversas ideas sostenidas y rechazadas por el profesorado, como si procedieran de una entrevista muy estructurada. El objetivo del análisis sería conectar ideas para estructurar el pensamiento global de una persona con sus puntos fuertes, sus puntos débiles, sus contradicciones y su coherencia.

\section{COMPARACIONES Y DIFERENCIAS ENTRE GRUPOS}

La cuantificación de las respuestas mediante los distintos índices actitudinales no sólo facilita los análisis diagnósticos anteriores, sino que también permite el contraste de hipótesis propio de la estadística inferencial. La investigación empírica plantea frecuentemente la necesidad de contrastar hipótesis basadas en comparar grupos: por ejemplo, para comprobar si un programa de educación CTS consigue el objetivo de cambiar las actitudes de los estudiantes en el sentido deseado o para decidir qué programa, entre varios, ofrece los mejores resultados. Estos asuntos requieren apli- car la estadística inferencial, que solamente es posible con una sólida base de datos cuantitativa. Como ilustración, se presentan en este apartado algunas aplicaciones de la metodología a la estadística inferencial en comparaciones de la muestra entre los subgrupos de hombres y mujeres y entre los subgrupos de titulación académica, empleando ANOVA y pruebas no paramétricas.

Se comparan aquí los resultados de actitudes CTS de los profesores y las profesoras; en primer lugar, para todos los índices medios de las frases incluidas en las cuestiones y, en segundo lugar, para los índices actitudinales globales de las cuestiones.

El centenar de comparaciones realizadas con los índices de cada una de las frases del cuestionario refleja escasas diferencias de género entre el profesorado. Solamente aparecen diferencias estadísticamente significativas en tres de ellas, aunque con niveles de significación poco intensos $(0,01<\mathrm{p}$ $<0,05)$. Las frases son la $\mathrm{D}$ (realización de experimentos) de la cuestión 10111 (definición de ciencia) ( $<<0,02$ ), la A (la ciencia no influye sobre la tecnología) de la cuestión 10412 (influencia de la ciencia sobre la tecnología) $(\mathrm{p}<0,04)$ y la $\mathrm{C}$ (los científicos interpretan los hechos de maneras distintas) de la cuestión 70211 (causas de las controversias científicas $)(p<0,035)$. Dado que la muestra de este estudio se encuentra en el límite de la estadística no paramétrica, se ha repetido el análisis anterior utilizando tests de significación propios de esta estadística (U de Mann-Whitney, W de Wilconson y Z), cuyos resultados son aún más restrictivos, pues solamente la frase $\mathrm{C}$ de la cuestión 70211 alcanza un nivel de significación apreciable $(\mathrm{p}<0,033)$. No obstante, los profesores obtienen índices de actitudes globales mejores que las mujeres (Tabla IX). 
Tabla IX

Resultados de la comparación de las actitudes CTS entre profesores y profesoras, y químicos y no-químicos de la muestra, entre los cuales no existen diferencias estadísticamente significativas.

\begin{tabular}{|c|c|c|c|c|c|c|}
\hline & & Adecuadas & Plausibles & Ingenuas & $\begin{array}{c}\text { Índice global } \\
\text { ponderado }\end{array}$ & $\begin{array}{l}\text { Índice } \\
\text { medio }\end{array}$ \\
\hline Mujeres & Media & ,2087 &,- 0565 &, 1484 & ,1002 & ,0651 \\
\hline$(\mathrm{N}=14)$ & Dv. est. &, 1634 &, 2717 & ,2497 & ,1118 & , 1508 \\
\hline$(\mathrm{N}=10)$ & Dv. est. & , 1296 & ,1859 & ,2044 & 0750 & ,1130 \\
\hline Mixto & Media & ,2463 &,- 0519 & ,1132 &, 1025 & 0590 \\
\hline$(\mathrm{N}=12)$ & Dv. est. &, 1534 &, 2705 & ,2364 &, 1122 &, 1470 \\
\hline
\end{tabular}

Las comparaciones entre el grupo de profesorado licenciado en ciencias químicas y el resto de la muestra tampoco ofrece diferencias significativas entre ambos. Los promedios de cada grupo se encuentran muy igualados (Tabla IX).

\section{CONCLUSIÓN}

Desde una perspectiva metodológica, los resultados obtenidos con la aplicación de esta nueva forma de evaluar las actitudes y creencias CTS del profesorado muestran la potencia de esta propuesta metodológica. Desde el punto de vista del diagnóstico, se pone en evidencia la inadecuación de las actitudes del profesorado en muchos temas CTS y, en consecuencia, la presumible falta de tratamiento adecuado de la educación científica en el aula; esta limitación queda patente mediante los índices de actitud obtenidos, que son positivos pero bajos.

El método de evaluación empleado permite no sólo cuantificar el grado de adecuación de las actitudes, personales, por cuestiones o por dimensiones y en contraste de hipótesis, sino también dejar abierta la perspectiva de realizar análisis cualitativos, tanto de los perfiles individuales de las personas como de las diversas dimensiones CTS o de cada cuestión particular. Este artículo explicita la nueva metodología empleada en los análisis, fundamentalmente los procesos cuantitativos, aunque se han dejado también referencias explícitas de la posibilidad de análisis cualitativos que no se exponen, pues su mera iniciación sería incompatible con la limitación del espacio disponible para este informe. La naturaleza interpretativa del análisis cualitativo se puede aplicar claramente en profundizar en el conocimiento de las actitudes, en discutir la validez y coherencia de las distintas respuestas de cada persona en cada cuestión o a través de distintas cuestiones o dimensiones, de manera similar a como se estudian las diversas respuestas abiertas de una entrevista para reconstruir la estructura de las actitudes clave del entrevistado. El análisis cualitativo queda evidentemente abierto a realizar las exploraciones y aportaciones típicas del mismo, con la ventaja del apoyo de los índices cuantitativos, que pueden actuar como pistas relevantes para guiar la reflexión interpretativa.

Para terminar, cabe recordar que, actualmente, la alfabetización científica y tecnológica para todas las personas debería ser la principal finalidad educativa de la enseñanza de las ciencias (AAAS, 1990, 1993; Acevedo, Manassero y Vázquez, 2002; Acevedo, Vázquez y Manassero, 2003; NRC, 1996) y una cuestión problemática para la didáctica de las ciencias por la dificultad de conseguirse (Gil y Vilches, 2001; Kemp, 2002). La multidimensionalidad de este concepto ofrece muchos matices para poder precisar las cualidades de una persona científicamente alfabetizada, de modo que no existe un acuerdo general sobre su significado (Bybee, 1997). Sin embargo, básicamente una persona alfabetizada debe ser capaz de alcanzar una comprensión de los principales conceptos, principios, teorías y procesos de la ciencia y una actitud positiva ante las complejas relaciones entre la ciencia, la tecnología y la sociedad (Acevedo, Manassero y Vázquez, 2002; Acevedo, Vázquez y Manassero, 2003). La metodología mostrada en este trabajo debe contribuir significativamente a evaluar este último componente esencial de la alfabetización, como es la actitud hacia la ciencia y la tecnología en su contexto social, que es el contenido fundamental del COCTS. 


\section{REFERENCIAS BIBLIOGRÁFICAS}

AAAS (1990). Science for all Americans. Nueva York: Oxford University Press.

AAAS (1993). Benchmarks for science literacy: A Project 2061 report. Nueva York: Oxford University Press.

ACEVEDO, J.A. (1996). La formación del profesorado de enseñanza secundaria y la educación CTS. Una cuestión problemática. Revista Interuniversitaria de Formación del Profesorado, 26, pp. 131-144. En línea en Sala de Lecturas CTS+I de la OEI, <http://www.campus-oei.org/salactsi/ acevedo9.htm>, 2001.

ACEVEDO, J.A. (2001). Una breve revisión de las creencias CTS de los estudiantes. En línea en Sala de Lecturas CTS+I de la OEI, <http://www.campus-oei.org/salactsi/ acevedo.htm>.

ACEVEDO, J.A., ACEVEDO, P., MANASSERO, M.A. y VÁZQUEZ, A. (2001). Avances metodológicos en la investigación sobre evaluación de actitudes y creencias CTS. En línea en Revista Iberoamericana de Educación, edición electrónica De los Lectores, <http://www.campus-oei.org/ revista/deloslectores/Acevedo.PDF $>$.

ACEVEDO, J.A., MANASSERO, M.A. y VÁZQUEZ, A. (2002). Nuevos retos educativos: hacia una orientación CTS de la alfabetización científica y tecnológica. Revista Pensamiento Educativo, 30, pp. 15-34.

ACEVEDO, J.A., VÁZQUEZ, A., ACEVEDO, P. y MANASSERO, M.A. (2002). Un estudio sobre las actitudes y creencias CTS del profesorado de primaria, secundaria y universidad. Tarbiya, 30, pp. 5-27. En línea en Sala de Lecturas CTS+I de la OEI, <http://www.campus-oei.org/ salactsi/acevedo15.htm>, 2003.

ACEVEDO, J.A., VÁZQUEZ, A. y MANASSERO, M.A. (2002). Evaluación de actitudes y creencias CTS: diferencias entre alumnos y profesores. Revista de Educación, 328, pp. 355-382. En línea en Sala de Lecturas CTS+I de la OEI, <http://www.campus-oei.org/salactsi/acevedo14.htm>, 2003.

ACEVEDO, J.A., VÁZQUEZ, A. y MANASSERO, M.A. (2003). Papel de la educación CTS en una alfabetización científica y tecnológica para todas las personas. Revista Electrónica de Enseñanza de las Ciencias, 2(2), artículo 1, $<\mathrm{http}: / /$ www.saum.uvigo.es/reec/>.

ACEVEDO, J.A., VÁZQUEZ, A., MANASSERO, M.A. y ACEVEDO, P. (2002a). Actitudes y creencias CTS de los alumnos: su evaluación con el Cuestionario de Opiniones sobre Ciencia, Tecnología y Sociedad. En línea en Revista Iberoamericana de Ciencia, Tecnología, Sociedad e Innovación, 2, <http://www.campus-oei.org/revistactsi/ numero2/varios $1 . h \mathrm{htm}>$.

ACEVEDO, J.A., VÁZQUEZ, A., MANASSERO, M.A. y ACEVEDO, P. (2002b). Persistencia de las actitudes y creencias CTS en la profesión docente. Revista Electrónica de Enseñanza de las Ciencias, 1(1). <http: //www.saum.uvigo.es/reec/>.

AIKENHEAD, G.S. (1987). High-school graduates' beliefs about science-technology-society. III. Characteristics and limitations of scientific knowledge. Science Education, 71(4), pp. 459-487.
AIKENHEAD, G.S. (1988). An analysis of four ways of assessing student beliefs about STS topics. Journal of Research in Science Teaching, 25(8), pp. 607-627.

AIKENHEAD, G.S. y RYAN, A.G. (1989). The development of a multiple choice instrument for monitoring views on ScienceTechnology-Society topics. Final Report of SSHRCC Grant. Saskatoon (Canadá): Department of Curriculum Studies, University of Saskatchewan.

AIKENHEAD, G.S. y RYAN, A.G. (1992). The development of a new instrument: «Views on science-technology-society»(VOSTS). Science Education, 76(5), pp. 477-491.

AIKENHEAD, G.S., RYAN, A.G. y DESAUTELS, J. (1989). «Monitoring student views on science-technology-society issues: The development of multiple-choice items». Comunication present Annual Meeting of the National Association for Research in Science Teaching. San Francisco (CA).

AIKENHEAD, G.S., RYAN, A.G. y FLEMING, R.W. (1989). Views on science-technology-society (from CDN.mc.5). Saskatoon (Canadá): Department of Curriculum Studies, University of Saskatchewan. En línea en <http: //www.usask.ca/education/people/aikenhead/vosts.pdf $>$.

AKERSON, V.L., ABD-EL-KHALICK, F. y LEDERMAN, N.G. (2000). Influence of a reflective explicit activity-based approach on elementary teachers' conceptions of nature of science. Journal of Research in Science Teaching, 37(4), pp. 295-317.

BYBEE, R.W. (1997). Achieving scientific literacy: From purposes to practices. Portsmouth $(\mathrm{NH})$. Heinemann.

CHO, J. (2002). The development of an alternative in-service programme for Korean science teachers with an emphasis on science-technology-society. International Journal of Science Education, 24(10), pp. 1021-1035.

FLEMING, R.W. (1987). High-school graduates' beliefs about science-technology-society. II. The interaction among science, technology and society. Science Education, 71(2), pp. 163-186.

FLEMING, R. (1988). Undergraduate science teachers' views on the relationship between science, technology and society. International Journal of Science Education, 10(4), pp. 449-463.

GARDNER, P.L. (1996). The dimensionality of attitude scales: A widely misunderstood idea. International Journal of Science Education, 18, pp. 913-919.

GIL, D. y VILCHES, A. (2001). Una alfabetización científica para el siglo XXI. Obstáculos y propuestas de actuación. Investigación en la Escuela, 43, pp. 27-37.

KEMP, A.C. (2002). Implications of diverse meanings for «scientific literacy». Comunicación presentada at the Annual International Conference of the Association for the Education of Teachers in Science. Charlotte (NC), en Rubba, P.A., Rye J.A., Di Biase, W.J. y Crawford, B.A. (eds.). Proceedings of the 2002 Annual International Conference of the Association for the Education of Teachers in Science, pp. 1202-1229. Pensacola (FL): AETS. En línea en <http: //www.ed.psu.edu/CI/Journals/2002aets/s3_kemp.rtf>. 
LEDERMAN, N.G. (1992). Students' and teachers' conceptions of the nature of science: A review of the research. Journal of Research in Science Teaching, 29(4), pp. 331-359.

LEDERMAN, N.G. y O'MALLEY, M. (1990). Students' perceptions of tentativeness in science: Development, use and sources of change. Science Education, 74(2), pp. 225-239.

MANASSERO, M.A. y VÁZQUEZ, A. (1998). Opinions sobre ciència, tecnologia $i$ societat. Palma de Mallorca: Govern Balear. Conselleria d'Educació, Cultura i Esports.

MANASSERO, M.A. y VÁZQUEZ, A. (1999). Ideas de los estudiantes sobre la epistemología de la ciencia: modelos, leyes y teorías. Revista de Educación, 309, pp. 309-334.

MANASSERO, M.A. y VÁZQUEZ, A. (2001). Opiniones sobre las relaciones entre ciencia, tecnología y sociedad. Tarbiya, 27, pp. 27-56.

MANASSERO, M.A., VÁZQUEZ, A. y ACEVEDO, J.A. (2001). Avaluació del temes de ciència, tecnologia $i$ societat. Palma de Mallorca: Conselleria d'Educació i Cultura.

MELLADO, V. (2003). Cambio didáctico del profesorado de ciencias experimentales y filosofía de la ciencia. Enseñanza de las Ciencias, 21(3) pp. 343-358.

NRC (1996). National Science Education Standards. Washington (DC): National Academic Press.

RUBBA, P.A. y HARKNESS, W.L. (1993). Examination of preservice and in-service secondary science teachers' beliefs about Science-Technology-Society interactions. Science Education, 77(4), pp. 407-431.

RYAN, A.G. y AIKENHEAD, G.S. (1992). Students' preconceptions about the epistemology of Science. Science Education, 76(6), pp. 559-580.

SHAPIRO, B.L. (1996). A case study of change in elementary student teacher thinking during an independent investigation in science: Learning about the «face of science that does not yet know». Science Education, 80(5), pp. 535-560.

SHRIGLEY, R.L. y KOBALLA Jr., T.R. (1992). A decade of attitude research based on Hovland's learning model. Science Education, 76(1), pp. 17-42.

SOLBES, J., VILCHES, A. y GIL, D. (2002). Formación del profesorado desde el enfoque CTS, en Membiela, P. (ed.). Enseñanza de las ciencias desde la perspectiva ciencia-tecnología-sociedad. Formación científica para la ciudadanía, pp. 163-175. Madrid: Narcea.

VÁZQUEZ, A., ACEVEDO, J.A. y MANASSERO, M.A. (2000). Progresos en la evaluación de actitudes relacionadas con la ciencia mediante el Cuestionario de Opiniones CTS, en Martins, I. P. (coord.). O Movimento CTS na Península Ibérica. Seminário Ibérico sobre Ciência-Tecnologia-Sociedade no ensino-aprendizagem das ciencias experimentais, pp. 219-230. Aveiro: Universidade de Aveiro. Versión electrónica en Sala de Lecturas CTS +I de la OEI. <http://www.campus-oei.org/salactsi/ acevedo6.htm (2001).

VÁZQUEZ, A. y MANASSERO, M.A. (1995). Actitudes relacionadas con la ciencia: una revisión conceptual. Enseñanza de las Ciencias, 13(3), pp. 337-346.

VÁZQUEZ, A. y MANASSERO, M.A. (1999). New response and scoring models for the «Views on Science-Technology-Society» instrument (VOSTS). International Journal of Science Education, 21(3), pp. 231-247.

ZOLLER, U., DONN, S., WILD, R. y BECKETT, P. (1991a). Students' versus their teachers' beliefs and positions on science-technology-society oriented issues. International Journal of Science Education, 13(1), pp. 25-35.

ZOLLER, U., DONN, S., WILD, R. y BECKETT, P. (1991b). Teachers' beliefs and views on selected science-technologysociety topics: A probe into STS literacy versus indoctrination. Science Education, 75(5), pp. 541-561. 